\title{
The Scale of Functional Specialization within Human Prefrontal Cortex
}

\author{
Sam J. Gilbert, ${ }^{1,2}$ Richard N. A. Henson, ${ }^{3}$ and Jon S. Simons ${ }^{4,5}$ \\ ${ }^{1}$ Institute of Cognitive Neuroscience and ${ }^{2}$ Division of Psychology and Language Sciences, University College London, London WC1N 3AR, United Kingdom, \\ ${ }^{3}$ Medical Research Council Cognition and Brain Sciences Unit, Cambridge CB2 7EF, United Kingdom, and ${ }^{4}$ Department of Experimental Psychology and \\ ${ }^{5}$ Behavioural and Clinical Neuroscience Institute, University of Cambridge, Cambridge CB2 3EB, United Kingdom
}

At what scale is it possible to observe consistent functional specialization within human prefrontal cortex (PFC), reproducible from one individual to the next? Some studies suggest gross functional divisions between large regions of PFC, but it is not known whether PFC exhibits specialization at the fine-grained scale known to differentiate posterior cortical functions. We used fMRI to confirm a three-way segregation of function between three regions of medial anterior PFC, each centered on coordinates within $15 \mathrm{~mm}$ of the other two. Naive participants performed three tasks based on earlier studies, and we investigated activity at regions defined by previous results. In each task, signal was significantly greater at the predicted region than the other two, just millimeters away. These results indicate reproducible functional specialization within PFC, at a much finer scale than previously demonstrated. Furthermore, these findings suggest that divergent results from previous studies may reflect the recruitment of functionally distinct regions and that "reverse inference" should be undertaken with caution.

\section{Introduction}

A fundamental principle of information processing in posterior cortical areas is specialization of distinct regions for different functions. This is well established in sensory regions such as occipital cortex, where a consistent patchwork of functionally distinct regions may be observed, demonstrated in the human brain by neuroimaging studies that describe separate maps of the visual field in adjacent cortical regions (Wandell et al., 2007). But what about prefrontal cortex (PFC), thought to be responsible for high-level behavioral regulation? Although some studies indicate gross functional divisions between large regions such as lateral versus orbital or dorsal versus ventral PFC, relatively little is known of its organization at the fine-grained scale known to differentiate posterior cortical functions.

Anatomical studies indicate that differences in cytoarchitecture and connectivity may exist on a similar scale within PFC as in sensory areas (Carmichael and Price, 1994; Beckmann et al., 2009). We therefore investigated whether we could find evidence of reproducible fine-grained functional specialization within $\mathrm{PFC}$, as the anatomical data might predict. Convincing evidence for functional specialization requires contrasting multiple tasks, performed by the same participants within the same experiment. Statistically, a region $\times$ contrast interaction, with regions defined

Received July 7, 2009; revised Nov. 5, 2009; accepted Nov. 23, 2009.

This research was supported by an award to J.S.S. via the University of Cambridge Behavioural and Clinical Neuroscience Institute, funded jointly by the UK Medical Research Council and the Wellcome Trust. S.J.G. was supported by a Royal Society University Research Fellowship and R.N.A.H. by the UK Medical Research Council (WBSE U.1055.05.012.00001.01). We thank Steve Eldridge and Helen Lloyd for invaluable scanning assistance.

Correspondence should be addressed to Sam Gilbert, Institute of Cognitive Neuroscience, 17 Queen Square, London WC1N 3AR, UK. E-mail: sam.gilbert@ucl.ac.uk.

DOI:10.1523/JNEUROSCI.3220-09.2010

Copyright $\odot 2010$ the authors $\quad 0270-6474 / 10 / 301233-05 \$ 15.00 / 0$ independently of the contrasts under consideration, would then be a minimum requirement to demonstrate functional specialization (Henson, 2006).

Some researchers argue that certain regions of PFC exhibit little intrinsic functional specialization (Duncan and Owen, 2000). However, one area of PFC that might be considered a likely candidate to exhibit fine-grained functional specialization is the medial anterior (or rostral) prefrontal cortex (MPFC), corresponding approximately to the medial aspects of Brodmann area 10, extending into medial aspects of Brodmann area 9 (Ramnani and Owen, 2004; Amodio and Frith, 2006; Burgess et al., 2007; Koechlin and Hyafil, 2007). Most evidence on the functions of this region comes from functional neuroimaging. However, this evidence has not yet converged on a coherent functional description of MPFC, with different studies attributing to the area disparate and seemingly incompatible roles. For example, three recent $\mathrm{fMRI}$ studies have implicated MPFC in (1) reflection on one's own emotions (Gusnard et al., 2001); (2) meta-memory processes, specifically recollection of internally generated rather than externally derived contextual details of prior stimuli (Simons et al., 2005); and (3) attention toward current perceptual rather than self-generated information (Gilbert et al., 2005).

These studies, investigating quite different tasks, produced peak MPFC coordinates very close to one another (each within 15 $\mathrm{mm}$ of the other two), perhaps suggesting common underlying processes. However, meta-analytic evidence (Gilbert et al., 2006) points toward the possibility of considerable fine-grained functional segregation within this region. The results of some neuroimaging studies may be consistent with this possibility (Gilbert et al., 2007; Krueger et al., 2007; Simons et al., 2008). However, without significant region $\times$ contrast interactions, based on independently defined regions, such studies remain preliminary 
and may be subject to statistical bias due to "double dipping" (Kriegeskorte et al., 2009). Here, we investigated empirically whether previous divergent results may reflect recruitment of functionally distinct regions within MPFC, potentially reconciling earlier findings.

\section{Materials and Methods}

We administered three tasks thought to recruit MPFC, based on earlier studies (Gusnard et al., 2001; Gilbert et al., 2005; Simons et al., 2005). For each task, we were able to define an independent region of interest (ROI), based on the coordinate of peak MPFC activation in the previous study. Signal was averaged over 6-mm-radius spheres centered on the previous peak voxel; this was the largest possible sphere before the ROIs began to overlap. Thus, for each participant in the present study, we extracted signal related to the three independent tasks, from three adjacent independently defined ROIs. In each task, we predicted a single ROI to be preferentially active, based on the previous results.

Participants. There were 16 participants (age: $20-32$ years; 8 male). All were healthy, right-handed, with normal or corrected-to-normal vision, and were not taking any psychoactive medication. All participants were screened using a comprehensive medical questionnaire and provided written informed consent before taking part. The study was approved by Cambridge Local Research Ethics Committee.

Tasks. Participants performed three tasks: emotion judgment (based on Gusnard et al., 2001), task memory (based on Simons et al., 2005), and perceptual attention (based on Gilbert et al., 2005).

In the emotion judgment task, participants were presented with 40 trials, each of which began with a fixation arrow indicating whether the stimulus word in the upcoming trial would be presented on the left or right of the monitor screen. After $500 \mathrm{~ms}$, a cue appeared at the bottom of the screen, indicating the task that was to be performed during that trial. Following a further $500 \mathrm{~ms}$, the stimulus word was presented in capital letters on the specified side of the screen. If the cue was " $1=$ natural $2=$ man-made," participants were instructed to decide whether the stimulus word was a naturally occurring or man-made object and press the appropriate key. If the cue was " $1=$ pleasant $2=$ unpleasant," participants made a judgment as to whether the stimulus word seemed pleasant or unpleasant to them. This is described as an emotion judgment task because participants were required to appraise a mental state as being positively or negatively valenced in this condition (Ochsner et al., 2004). Participants were given $2 \mathrm{~s}$ to make their judgment and were instructed before the scanning session to remember the position of the stimulus words, and the task they performed on them, for a subsequent memory test. The intertrial interval was jittered according to an exponential distribution between 480 and $1080 \mathrm{~ms}$.

In the task memory task, participants' memories were tested for the 40 stimulus words encountered in the preceding emotion judgment phase. A cue was first presented at the bottom of the screen, indicating the recollective judgment participants were to make on the stimulus word in the upcoming trial. After $500 \mathrm{~ms}$, the stimulus (a word that had been presented during the preceding emotion judgment phase) appeared in the center of the screen. If the cue was " $1=$ natural $/$ man-made $2=$ pleasant/unpleasant," participants tried to remember which of the two tasks they had performed with the stimulus word during the preceding phase. If the cue was " $1=$ left $2=$ right," participants tried to remember whether the stimulus word had been presented on the left or the right of the screen in the preceding phase. Participants had $2.5 \mathrm{~s}$ to make their judgment, and the intertrial interval jittered between 480 and $1080 \mathrm{~ms}$ as above.

In the perceptual attention task, participants alternated between (1) stimulus-oriented ( $\mathrm{SO}$ ) phases, where they responded to visually presented information, and (2) stimulus-independent (SI) phases, where they responded to self-generated information. During SO phases, participants were presented on each trial with a row of identical capital letters. The number of letters was matched to the stimuli in the emotion judgment and task memory tasks. Participants were asked to press one of two buttons to indicate whether the letters on the screen were composed entirely of straight lines (e.g., "A") or contained a curve (e.g., "B"). Im- mediately following each response, the next letter in the alphabet was presented, returning to the letter A after Z. In the SI phase, participants were asked to mentally continue the alphabet sequence and continue pressing buttons as before; the screen displayed alternating question marks and upside-down question marks on each trial. This ensured that the two phases were matched in that responses were always accompanied by a visual change. Transitions between the two phases occurred at unpredictable times (uniform distribution, 3.1-18.8 s, mean: $10.9 \mathrm{~s}$ ).

These tasks were alternated with a filler task in which participants made left and right key presses alternately to make a row of nine "X"s flip as quickly as possible between a horizontal and vertical orientation. The stimulus was immediately removed from the screen after each keypress, followed by a random delay between 300 and $700 \mathrm{~ms}$ to induce participants to pay attention to the stimuli. This task [based on the baseline condition of Simons et al. (2006)] was included to provide a break between the three experimental tasks of interest. This ensured that the task memory task did not follow on immediately from the preceding emotion judgment task from which its stimuli were drawn, minimizing rehearsal of individual items.

Procedure. Instructions for the tasks were first presented to participants in a 15 min practice session, during which they practiced each task until familiar with the requirements. In the scanner, participants underwent four runs of $\sim 10$ min each. Each task was performed once within each run for $\sim 2.5 \mathrm{~min}$, followed by $30 \mathrm{~s}$ of the filler task. Tasks began with a screen announcing which task was to be performed and instructing participants to get ready, displayed for $4 \mathrm{~s}$. There were two possible task orders, assignment of which was counterbalanced between participants: (1) emotion judgment, (2) task memory, (3) perceptual attention; or (1) perceptual attention, (2) emotion judgment, (3) task memory. The stimuli for the task memory task were always drawn from the immediately preceding emotion judgment task, necessitating a fixed order of these two tasks within each run.

Scanning procedure. A 3T Siemens TIM Trio system was used to acquire structural and echo-planar functional images $(\mathrm{TR}=2.25 \mathrm{~s} ; \mathrm{TE}=30 \mathrm{~ms} ; 36$ sequential axial slices oriented $\sim 10-20^{\circ}$ to the AC-PC transverse plane, 2 mm thickness, $1 \mathrm{~mm}$ interslice skip; $3 \mathrm{~mm} \times 3 \mathrm{~mm}$ in-plane resolution, $64 \times$ 64 pixels; $78^{\circ}$ flip angle; 4 sessions each of 250 volume acquisitions). The first six volumes from each session were discarded to allow for T1 equilibration.

Data analysis. fMRI data were analyzed using SPM8 software (http://www.fil.ion.ucl.ac.uk/spm/software/spm8/). The volumes were realigned, corrected for different slice acquisition times, normalized into $3 \mathrm{~mm}$ cubic voxels using the Montreal Neurological Institute (MNI) reference brain using fourth-degree B-spline interpolation, and smoothed with an isotropic $8 \mathrm{~mm}$ full-width half-maximum Gaussian kernel. A separate analysis was additionally conducted omitting the smoothing step.

The volumes acquired during the four sessions were treated as separate time series. For each series, the variance in the BOLD signal was decomposed with a set of regressors in a general linear model. Separate regressors coded for trial onsets in the following conditions: (1) trials of the filler task; (2) stimulus-oriented trials in the perceptual attention task; (3) stimulus-independent trials in the perceptual attention task; (4) pleasantness judgments in the emotion judgment task; (5) living/nonliving judgments in the emotion judgment task; (6) correct task recollection in the task memory task; (7) correct position recollection in the task memory task; and (8) error trials in the task memory task. Each regressor was generated with delta functions corresponding to each trial onset convolved with a canonical hemodynamic response function. These regressors, together with the regressors representing residual movementrelated artifacts and the mean over scans, comprised the full model for each session. The data and model were high-pass filtered to a cutoff of $1 / 128 \mathrm{~Hz}$.

Parameter estimates for each regressor were calculated from the least mean squares fit of the model to the data. Three linear contrasts were performed for each effect of interest, separately for each participant, i.e., perceptual attention ( 2 vs 3 ; see list of regressors above), emotion judgment (4 vs 5 ), and task memory (6 vs 7 ). Thus each task had its own separate experimental and control conditions, from which contrast estimates were calculated. This ensured that experimental and control con- 


\section{Regions of interest from previous studies}

Region 1

Gusnard et al. (2001):

Emotion judgment

$-3,53,24$

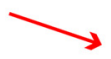

Region 2

Task memory

$-9,63,21$

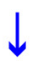

Simons et al. (2005):
Region 3

Gilbert et al. (2005):

Perceptual attention

$0,64,26$

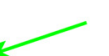

Adjacent 6mm-radius spheres

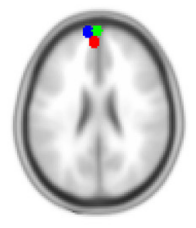

$z=24$

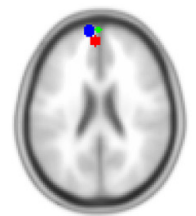

$z=21$

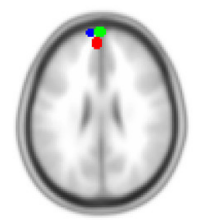

$z=26$

\section{Present study (within-subjects design)}
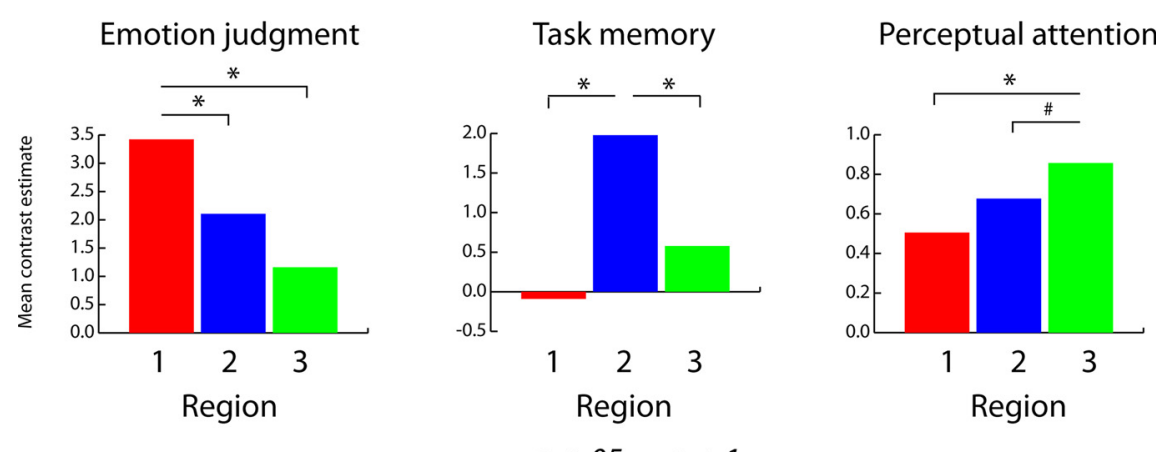

$$
* p<.05 \# p<.1
$$

Figure 1. Illustration of the three regions of interest and mean contrast estimates in each region, plotted separately for each task. Note that timing parameters were not comparable in the three tasks, and hence it is not possible to make direct comparisons between parameter estimates across different tasks. Crucially, however, a different pattern of parameter estimates across the three regions was observed within each task. These results confirm the predicted three-way segregation of function between three adjacent regions within anterior medial prefrontal cortex.

ditions were matched for each task (e.g., in terms of visual stimulation), so that the contrast estimates would reflect as far as possible the psychological processes of interest.

\section{Results}

\section{Behavioral data}

\section{Emotion judgment}

Mean accuracy was $94 \%$ in the control living/nonliving judgment condition. Because the pleasantness judgment condition required a subjective pleasant/unpleasant response it was not possible to calculate accuracy in this condition. Mean response times (RTs) were $1106 \mathrm{~ms}$ in the living/nonliving judgment condition and $1176 \mathrm{~ms}$ in the pleasantness judgment condition $\left(t_{(15)}=4.2\right.$, $p<0.001)$.

\section{Task memory}

Mean accuracy was $72 \%$ in the control position judgment condition and $80 \%$ in the task judgment condition $\left(t_{(15)}=2.7\right.$, $p=0.016)$. Mean RTs were $1286 \mathrm{~ms}$ in the position judgment condition and 1511 in the task judgment condition $\left(t_{(15)}=\right.$ 6.6, $p<0.001)$.

\section{Perceptual attention}

Mean accuracy was $97 \%$ in the control SI condition and $97 \%$ in the SO condition $\left(t_{(15)}=0.26, p=0.8\right)$. Mean RTs were 548 $\mathrm{ms}$ in the SI condition and $525 \mathrm{~ms}$ in the SO condition $\left(_{(15)}=\right.$ $2.9, p=0.01)$.

\section{Neuroimaging data}

All three tasks in the present study produced significant activation at their predicted ROI, compared with their respective control conditions (emotion judgment: $t_{(15)}=$ $3.5, p=0.0016$; task memory: $t_{(15)}=2.3$, $p=0.018$; perceptual attention: $t_{(15)}=$ 4.2, $p=0.0004)$, replicating previous findings. Furthermore, for each of the three tasks, activity was greater at the predicted ROI than the other two ROIs. This was confirmed statistically by comparing activity in the predicted ROI with the mean activity of the other two ROIs in each task (emotion judgment: $t_{(15)}=2.6$, $p=0.011$; task memory: $t_{(15)}=2.5, p=$ 0.013; perceptual attention: $t_{(15)}=2.3$, $p=0.017$; see Fig. 1 for all pairwise comparisons). Thus, within each task, the ROI yielding greatest activation was the one predicted from previous studies, and the difference between the most active ROI and the average of the other ROIs was significant in each case.

We conducted an additional analysis in which we contrasted the three experimental conditions against the shared visuomotor filler task rather than their respective control conditions. In the emotion judgment contrast, activity was greater at the predicted ROI than the other ROIs $\left(t_{(15)}=\right.$ $2.15, p<0.05)$. Likewise for the task memory contrast $\left(t_{(15)}=6.34, p<0.001\right)$. However, there was no longer a significant effect for the perceptual attention contrast $\left(t_{(15)}=0.41, p=0.69\right)$. This is consistent with the idea that the visuomotor task also involved a strong degree of stimulusoriented attention, making it a poor control condition for the perceptual attention contrast, thus underlining the importance of individually matching each experimental condition with its own control.

To confirm the present evidence for functional specialization, we conducted repeated-measures ANOVAs to investigate the region $\times$ contrast interactions. For these analyses, the 48 data points for each task (i.e., 16 participants $\times 3$ ROIs) were first converted into $z$ scores. This ensures that results cannot simply be driven by overall differences in activity yielded by the three tasks. However, all results below were statistically equivalent when untransformed contrast estimates were used. In the $3 \times 3$ ANOVA including all tasks and regions, the region $\times$ contrast interaction was significant $\left(F_{(4,12)}=3.9, p=0.029\right)$. Furthermore, all three $2 \times 2$ ANOVAs investigating predicted interactions between pairs of contrasts and associated regions were also significant (emotion judgment vs task memory: $F_{(1,15)}=10.5, p=0.005$; task memory vs perceptual attention: $F_{(1,15)}=5.8, p=0.030$; perceptual attention vs emotion judgment: $F_{(1,15)}=17.0, p<$ $0.001)$. All neuroimaging results were qualitatively similar when unsmoothed data were used.

Region $\times$ contrast interactions such as these could result, trivially, from vascular steal (where a BOLD signal increase in one region causes a BOLD signal decrease in a nearby region, even though the neural activity in the latter region is unchanged), or 
from regions that are reciprocally interconnected (where increased neural activity in one region causes decreased neural activity in another) (Henson, 2006). However, both of these possibilities are ruled out by the finding that all three regions showed simultaneous increases in BOLD signal under some contrasts, e.g., between SO and SI conditions of the perceptual attention task $\left(t_{(15)}>3.8, p<0.002\right)$. Although the significant two-way interactions were not cross-over interactions in every pairwise comparison of regions and tasks, under the assumption that the neural-BOLD mapping is identical across the three regions (which seems reasonable given their proximity in terms of local vasculature), the present results would then meet the criteria set out by Henson (2006) for a qualitative difference in brain activity.

\section{Discussion}

The present results confirm a predicted three-way segregation of function between three adjacent ROIs within MPFC, all centered on coordinates falling within $15 \mathrm{~mm}$ of each other. These findings provide robust within-subjects evidence confirming reproducible fine-grained functional specialization within MPFC. Thus, at least within certain regions of human prefrontal cortex, functional specialization may be observed on a similar scale to that observed in early sensory regions. Note that the experimental condition was associated with significantly slower RTs than the control condition in the emotion judgment and task memory tasks, but significantly faster RTs than the control condition in the perceptual attention task. Thus, the present results cannot reflect differences in time-on-task, or "difficulty" (as indexed by RT) between conditions, seeing as opposite patterns of RTs were observed across tasks, despite all three tasks being associated with a consistent direction of signal change in MPFC.

The question of functional specialization within a particular brain region may be approached at different levels of description (Henson, 2005). For example, at one level of description, occipital cortex may be described as supporting vision. At a finer level of description, different parts of occipital cortex may be distinguished according to their sensitivity to different types of visual information (motion, color, etc.); at a finer level still, different parts may be distinguished according to their sensitivity to different portions of the visual field (Wandell et al., 2007). At a coarse level of description, MPFC may be involved in biasing attention between self-generated and externally derived information (Burgess et al., 2007). However, at a finer level of description, the present results may help to resolve divergent findings from earlier studies. For example, it is hard to reconcile the suggestion that MPFC supports attention toward perceptual rather than selfgenerated information (Gilbert et al., 2005) with evidence that this region is involved in recalling internally rather than externally generated contextual information associated with an earlier stimulus (Simons et al., 2005). Our results suggest that divergent results such as these could reflect the recruitment of functionally distinct regions in different studies.

It is an open question how far these functional distinctions reflect different intrinsic computations and how far they reflect similar general processes operating on different input and output representations. This is particularly the case seeing as the tasks investigated in the present study, and most other studies of highlevel cognition, are relatively poorly understood at an informationprocessing level. However, evidence from neuroanatomy would be consistent with the idea that the functional distinctions observed in this study arise, at least in part, from different patterns of connectivity within MPFC. For example, Porrino et al. (1981) found that connections between the amygdala and medial pre- frontal cortex of the rhesus monkey are more dense in the anterior cingulate than the frontal pole. This would be consistent with the finding that a relatively posterior area within MPFC was particularly involved in the emotion judgment task in the present study. The present results would also be consistent with the larger-scale organization of rostral prefrontal cortex identified in the meta-analysis of Gilbert et al. (2006). In this meta-analysis, a relatively posterior medial region was implicated in mentalizing studies (i.e., those involving reflection on one's own mental states or those of another person), particularly when they involved emotional materials. In contrast, relatively lateral regions were implicated in studies involving episodic memory retrieval, as in the task memory task administered in the present study.

Despite the present evidence for functional specialization, some neuropsychological studies report surprisingly little impairment even in patients with extensive medial frontal lesions (e.g., Bird et al., 2004). There are at least two possible explanations for this divergence between neuroimaging and neuropsychological results. First, even though different brain regions may be preferentially recruited by different types of task, there may nevertheless be considerable redundancy in the brain mechanisms that support particular abilities. For example, in the present study, even though the three regions were preferentially activated by different tasks, all three regions generally responded with a consistent direction of signal change in all three tasks (i.e., greater for the experimental than the control condition). A second possible explanation for the divergence between neuroimaging and neuropsychological findings is that the tests used for neuropsychological assessment are relatively insensitive to the abilities supported by MPFC [see Burgess et al. (2009) for discussion of this point].

Along with theoretical implications for our understanding of the role of MPFC, the present results have methodological implications for the study of cognitive architecture using fMRI. Because the three regions investigated in this study are relatively close to one another, the present findings caution against the use of fMRI results to link activity in a particular brain area with engagement of a specific cognitive process (i.e., "reverse inference") (Poldrack, 2006). Rather than striving to identify a single common process supported by MPFC, a more fruitful approach may be to consider this area as comprising multiple, functionally distinct subregions, similar to the organization known to exist in posterior cortex. Such an approach is compatible not only with the functional neuroimaging data, but fits also with evidence of distinct connectivity networks from studies in humans and nonhuman primates (Amodio and Frith, 2006; Beckmann et al., 2009).

\section{References}

Amodio DM, Frith CD (2006) Meeting of minds: the medial frontal cortex and social cognition. Nat Rev Neurosci 7:268-277.

Beckmann M, Johansen-Berg H, Rushworth MFS (2009) Connectivitybased parcellation of human cingulate cortex and its relation to functional specialization. J Neurosci 29:1175-1190.

Bird CM, Castelli F, Malik O, Frith U, Husain M (2004) The impact of extensive medial frontal lobe damage on 'theory of mind' and cognition. Brain 127:914-928.

Burgess PW, Dumontheil I, Gilbert SJ (2007) The gateway hypothesis of rostral prefrontal cortex (area 10) function. Trends Cogn Sci 11:290-298.

Burgess PW, Alderman N, Volle E, Benoit RG, Gilbert SJ (2009) Mesulam's frontal lobe mystery re-examined. Restor Neurol Neurosci 27:493-506.

Carmichael ST, Price JL (1994) Architectonic subdivision of the orbital and medial prefrontal cortex in the macaque monkey. J Comp Neurol 346:366-402. 
Duncan J, Owen AM (2000) Common regions of the human frontal lobe recruited by diverse cognitive demands. Trends Neurosci 23:475-483.

Gilbert SJ, Frith CD, Burgess PW (2005) Involvement of rostral prefrontal cortex in selection between stimulus-oriented and stimulus-independent thought. Eur J Neurosci 21:1423-1431.

Gilbert SJ, Spengler S, Simons JS, Steele JD, Lawrie SM, Frith CD, Burgess PW (2006) Functional specialization within rostral prefrontal cortex (area 10): a meta-analysis. J Cogn Neurosci 18:932-948.

Gilbert SJ, Williamson ID, Dumontheil I, Simons JS, Frith CD, Burgess PW (2007) Distinct regions of medial rostral prefrontal cortex supporting social and non-social functions. Soc Cogn Affect Neurosci 2:217-226.

Gusnard DA, Akbudak E, Shulman GL, Raichle ME (2001) Medial prefrontal cortex and self-referential mental activity: relation to a default mode of brain function. Proc Natl Acad Sci U S A 98:4259-4264.

Henson RN (2005) What can functional imaging tell the experimental psychologist? Q J Exp Psychol A 58:193-233.

Henson RN (2006) Forward inference in functional neuroimaging: dissociations vs associations. Trends Cogn Sci 10:64-69.

Koechlin E, Hyafil A (2007) Anterior prefrontal function and the limits of human decision-making. Science 318:594-598.

Kriegeskorte N, Simmons WK, Bellgowan PSF, Baker CI (2009) Circular analysis in systems neuroscience: the dangers of double dipping. Nat Neurosci 12:535-540.

Krueger F, Moll J, Zahn R, Heinecke A, Grafman J (2007) Event frequency modulates the processing daily life activities in human medial prefrontal cortex. Cereb Cortex 17:2346-2353.

Ochsner KN, Knierim K, Ludlow DH, Hanelin J, Ramachandran T, Glover G, Mackey SC (2004) Reflecting upon feelings: an fMRI study of neural systems supporting the attribution of emotion to self and other. J Cogn Neurosci 16:1746-1772.

Poldrack RA (2006) Can cognitive processes be inferred from neuroimaging data? Trends Cogn Sci 10:59-63.

Porrino LJ, Crane AM, Goldman-Rakic PS (1981) Direct and indirect pathways from the amygdala to the frontal lobe in rhesus monkeys. J Comp Neurol 198:121-136.

Ramnani N, Owen AM (2004) Anterior prefrontal cortex: insights into function from anatomy and neuroimaging. Nat Rev Neurosci 5:184-194.

Simons JS, Owen AM, Fletcher PC, Burgess PW (2005) Anterior prefrontal cortex and the recollection of contextual information. Neuropsychologia 43:1774-1783.

Simons JS, Schölvinck ML, Gilbert SJ, Frith CD, Burgess PW (2006) Differential components of prospective memory? Evidence from fMRI. Neuropsychologia 44:1388-1397.

Simons JS, Henson RN, Gilbert SJ, Fletcher PC (2008) Separable forms of reality monitoring supported by anterior prefrontal cortex. J Cogn Neurosci 20:447-457.

Wandell BA, Dumoulin SO, Brewer AA (2007) Visual field maps in human cortex. Neuron 56:366-383. 